\title{
Analysis Method on Network Capacity of Belt Freeway
}

\author{
Shengwen TUa, Junjie CHEN and Yao ZHANG \\ School of Material and Architecture Engineering,Guizhou Normal University,Guiyang,China
}

\begin{abstract}
An analysis method on network capacity of belt freeway based on road network reserve capacity is put forward in this paper. Through the introduction of multiple multiplier, the method can simply analyse the maximum traffic volume contained by belt freeway in the conditions of a given network basic demand and meeting the constraints of road traffic capacity and service level. In comparison to the traditional method, the proposed method takes into consideration actual network structure, OD flow distribution, the traveller choosing the way, etc. Additionally, the analysis conclusion is more consistent with the actual situation. Analysis results of belt freeway capacity of Yancheng city in China show that the presented method is not only simple and practical, but also can objectively reflect the status of the belt freeway capacity, and provide a basis for decision-making of belt freeway expansion and urban expressway construction.
\end{abstract}

\section{Introduction}

The belt freeway has an important position in the grouptype urban for connecting traffic organization, almost all through transportation need to pass the belt freeway to realize direction change, parts of entry and exit traffic transportation need to go through the belt freeway for evacuation, parts of long distance transportation between groups can reach the destination through the belt freeway; therefore, traffic volume of most urban belt freeway is quite concentrated, with heavy traffic load. How to conduct quantitative analysis of belt freeway capacity is the real problem faced by planners and decision makers.

The capacity of transportation network means the maximum space transfer capacity of people and objects provided by the entire transportation network within the limits of urban space and given the constraints of transportation facilities resources and environmental capacity, which is also known as the capacity of road network or traffic capacity of road network [1].Scholars at home and abroad[2-7] have done a lot of researches on analysis calculation of the capacity of road network or traffic capacity of road network, and its main methods are space-time consumption method, linear programming method, cut set method, traffic distribution simulation method, narrow network capacity analysis method, etc. The traditional network capacity calculation model usually regards road network as a simple physical network, only considering the influence of road traffic capacity on the whole network capacity, but ignoring actual structure of road network and the road selection behaviour of users.

\section{Analysis Method of Belt Freeway Capacity Based on Reserve Capacity of Road Network}

In recent years, the concept of road network reserve capacity has aroused a lot of scholars to study capacity of the road network. Given the basic demand of road network $q_{r s}(r \in R, s \in S, R$ and $S$ belong to O、D set), under the restrictions of meeting road section capacity and service level, if road network can hold the maximum traffic $\mu \cdot q_{r s}$, it will be deemed that the multiple multiplier $\mu$ is reserve capacity of road network. The reserve capacity shows that the adaption of road network to the capacity of a given traffic demand structure; the multiple multiplier $\mu>1$ shows that most of current networks have the surplus capacity exceeding the current traffic demand $(\mu-1)$ times; the multiple multiplier $\mu<1$ shows that network load has overloaded, surpassing the current traffic demand $(1-\mu)$ times.

Usually, the problem for solving the network reserve capability can be described by the following bi-level programming problem [6-7].

$$
\begin{array}{ll} 
& \operatorname{maximize} \quad \mathrm{Z}=\mu \\
\text { s.t. } & v_{a}(\mu) \leq \rho_{a} C_{a}, \forall a \in A
\end{array}
$$

Where $A$ is the set of links, $\rho_{a}$ is a parameter denoting the pre-specified LOS required on link $A, C_{a}$ is the capacity of link $a, v_{a}(\mu)$ is the flow on link $a$, which is obtained by solving the lower-lower user equilibrium (UE) assignment problem under a given reserve capacity multiplier $\mu$,

$$
\operatorname{minimize} \sum_{a \in A} \int_{0}^{v_{a}} t_{a}(w) d w
$$

\footnotetext{
a Corresponding author: 79418129@qq.com
} 


$$
\begin{array}{cl}
\text { s.t. } \quad & \sum_{k \in K_{r s}} f_{k}^{r s}=\mu \cdot q_{r s}, \forall r \in R, s \in S \\
v_{a}= & \sum_{r \in R} \sum_{s \in S} \sum_{k \in K_{r s}} f_{k}^{r s} \delta_{a k}^{r s}, \forall a \in A \\
f_{k}^{r s} \geq 0, \forall k \in K_{r s}, r \in R, s \in S
\end{array}
$$

Where $R$ and $S$ are respectively the sets of origins and destinations, $t_{a}$ is the travel time on link $a, f_{k}^{r s}$ is the flow on route $k$ between O-D pair $(r, \mathrm{~s}), \delta_{a k}^{r s}$ is the link-route incidence indicator, $\delta_{a k}^{r s}=1$ if link $a$ is on route $k$ between O-D pair $(r, s), \delta_{a k}^{r s}=0$ otherwise. In this formulation, the objective function Eq.(1) is to maximize the multiplier $\mu$, Eq.(2) is the link LOS constraint,Eq.(4) is the demand conservation constraint,Eq.(5) is a definitional constraint that sums up all route flows that pass through a given link, and Eq.(6) is a non-negativity constraint on the route flows.

The researches of XU[8], ZHU[9] et al. show that the reserve capacity of road network not only considers the structure of traffic demand, but also takes into account the congestion effect of road network and the road selection behavior of users, so it is more suitable to evaluate the actual capacity of the road network.

This paper applies the analysis method of reserve capacity to study the belt freeway capacity status. Because the belt freeway is a ring network, the path to be chosen by any traveller is only two paths in two directions, i.e. a positive direction and a negative direction. Usually, the traveller chooses the shorter path; therefore, there is no need for the analysis of the users' equilibrium assignment, and the bi-level programming problem Eq.(1)-Eq. (6) can be simplified as the following simple linear programming problem.

$$
\begin{gathered}
\text { maximize } \quad Z=\mu \\
\text { s.t. } \quad v_{a}(\mu) \leq \rho_{a} C_{a}, \forall a \in A \\
\sum_{k \in K_{r s}} f_{k}^{r s}=\mu \cdot q_{r s}, \forall r \in R, s \in S \\
v_{a}=\sum_{r \in R} \sum_{s \in S} \sum_{k \in K_{r s}} f_{k}^{r s} \delta_{a k}^{r s}, \forall a \in A \\
f_{k}^{r s} \geq 0, \forall k \in K_{r s}, r \in R, s \in S
\end{gathered}
$$

The meaning of the variable is the same as that mentioned above, where Eq. (7) is the objective function and the Eq. (8) -Eq. (11)is the constraint condition. If the present situation and the planned traffic distribution of each node on the belt freeway and the technical standard of the belt freeway are provided, the current situation of the belt freeway or the reserve capacity of the planned year can be simply analyzed using Eq.(7)-Eq.(11)to determine the capacity status of the belt freeway.

\section{Numerical Results}

\subsection{Road Network Model}

The application of the above method is illustrated by the analysis of the capacity of the belt freeway of Yancheng, Jiangsu Province. Figure 1 is the layout of arterial highways around Yancheng city, which consists of many freeways, national highways and provincial highways. Each section of Yancheng belt freeway is a two-way four-lane highway with a design speed of $100 \mathrm{~km} / \mathrm{h}$ and a total length of $83.5 \mathrm{~km}$. Figure 2 shows the abstract road network model of the belt freeway.
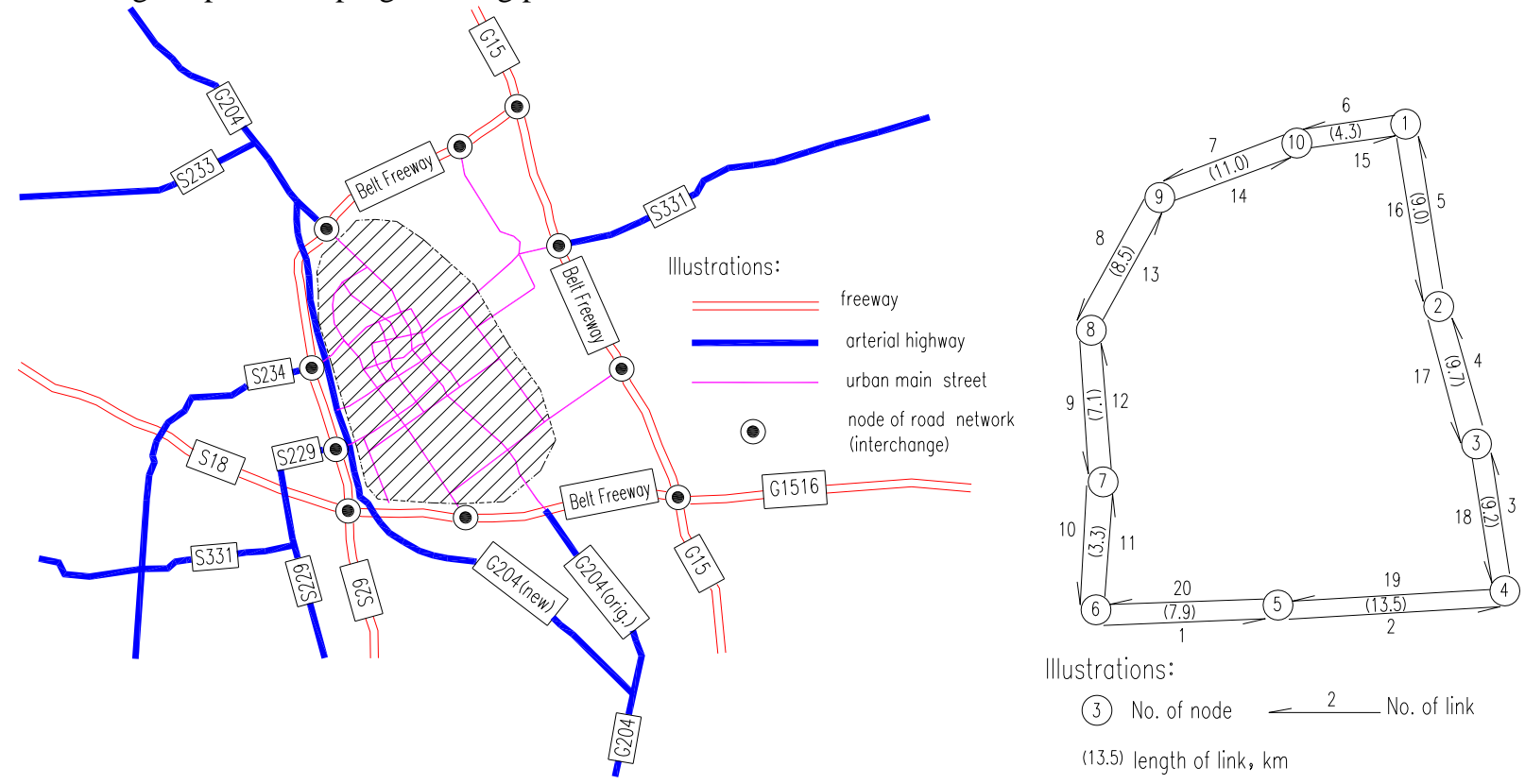

Figure.1 The Layout of Arterial Highway Around Yancheng City

Figure.2 The Network Model of Belt Freeway of Yancheng 
OD survey data of 2012 and forecast data of 2025 are shown in Table 1 and Table 2 respectively. The design service level of each section of the belt freeway is considered as level 3, that is, $\rho_{a}=0.65$. The basic capacity of single-lane freeway is $2000 \mathrm{pcu} / \mathrm{h}$, and the coefficient of two-lane in one driving direction is 1.87 , then the basic capacity of one driving direction is $3740 \mathrm{pcu} / \mathrm{h}$. Peak hour flow ratio is taken $10 \%$.

Table 1. The OD Survey Data Between Road Network Nodes in 2012 (pcu/d)

\begin{tabular}{|c|c|c|c|c|c|c|c|c|c|c|}
\hline \multirow{2}{*}{ Orig. } & \multicolumn{10}{|c|}{ Destination } \\
\cline { 2 - 11 } & 1 & 2 & 3 & 4 & 5 & 6 & 7 & 8 & 9 & 10 \\
\hline 1 & 0 & 1023 & 339 & 7742 & 378 & 4514 & 558 & 609 & 813 & 742 \\
\hline 2 & 983 & 0 & 327 & 838 & 354 & 2426 & 307 & 329 & 609 & 103 \\
\hline 3 & 359 & 337 & 0 & 862 & 103 & 2317 & 425 & 389 & 359 & 113 \\
\hline 4 & 7653 & 848 & 813 & 0 & 1228 & 5287 & 508 & 327 & 409 & 106 \\
\hline 5 & 368 & 345 & 105 & 1218 & 0 & 772 & 108 & 97 & 229 & 89 \\
\hline 6 & 4505 & 2208 & 2435 & 5247 & 802 & 0 & 902 & 708 & 819 & 335 \\
\hline 7 & 549 & 327 & 416 & 498 & 107 & 922 & 0 & 249 & 349 & 101 \\
\hline 8 & 634 & 349 & 389 & 307 & 99 & 678 & 229 & 0 & 329 & 231 \\
\hline 9 & 887 & 689 & 359 & 409 & 209 & 819 & 359 & 309 & 0 & 344 \\
\hline 10 & 721 & 105 & 102 & 109 & 87 & 341 & 97 & 228 & 351 & 0 \\
\hline
\end{tabular}

Table 2. The OD Forecasting Data Between Road Network Nodes in 2025 (pcu/d)

\begin{tabular}{|c|c|c|c|c|c|c|c|c|c|c|}
\hline \multirow{2}{*}{ Orig. } & \multicolumn{10}{|c|}{ Destination } \\
\cline { 2 - 12 } & 1 & 2 & 3 & 4 & 5 & 6 & 7 & 8 & 9 & 10 \\
\hline 1 & 0 & 1193 & 396 & 9055 & 440 & 5283 & 641 & 703 & 960 & 964 \\
\hline 2 & 1146 & 0 & 385 & 9991 & 415 & 2841 & 359 & 385 & 713 & 121 \\
\hline 3 & 411 & 394 & 0 & 1009 & 121 & 2711 & 497 & 455 & 420 & 132 \\
\hline 4 & 8954 & 992 & 951 & 0 & 1437 & 6186 & 594 & 383 & 479 & 124 \\
\hline 5 & 431 & 404 & 123 & 1425 & 0 & 903 & 126 & 113 & 268 & 104 \\
\hline 6 & 5271 & 2583 & 2849 & 6144 & 938 & 0 & 1055 & 828 & 958 & 392 \\
\hline 7 & 642 & 383 & 487 & 583 & 125 & 1079 & 0 & 291 & 408 & 118 \\
\hline 8 & 742 & 408 & 455 & 359 & 116 & 793 & 268 & 0 & 385 & 274 \\
\hline 9 & 1038 & 806 & 431 & 479 & 245 & 956 & 420 & 362 & 0 & 402 \\
\hline 10 & 844 & 126 & 121 & 128 & 102 & 399 & 113 & 267 & 411 & 0 \\
\hline
\end{tabular}

According to the data of OD of 2012 in Table 1, and substituting the above parameters into Eq.(7)-Eq.(11), the multiple multiplier $\mu$ of belt freeway in 2012 is calculated to be 1.17 , and the $V / C$ value of each link is shown in Table 3. Similarly, the multiple multiplier $\mu$ of belt freeway in 2025 is calculated to be 0.92 , and the $V / C$ value of each link is shown in Table 4.

Table 3. V/C Value of Links (in 2012)

\begin{tabular}{|c|c|c|c|c|c|c|c|c|c|c|}
\hline No. of link & 1 & 2 & 3 & 4 & 5 & 6 & 7 & 8 & 9 & 10 \\
\hline V/C & 0.42 & 0.49 & 0.56 & 0.51 & 0.48 & 0.15 & 0.13 & 0.13 & 0.13 & 0.13 \\
\hline \hline No. of link & 11 & 12 & 13 & 14 & 15 & 16 & 17 & 18 & 19 & 20 \\
\hline V/C & 0.09 & 0.08 & 0.12 & 0.12 & 0.12 & 0.12 & 0.15 & 0.48 & 0.51 & 0.56 \\
\hline
\end{tabular}

Table 4. V/C Value of Links (in 2025)

\begin{tabular}{|c|r|r|r|r|r|r|r|r|r|r|}
\hline No. of link & 1 & 2 & 3 & 4 & 5 & 6 & 7 & 8 & 9 & 10 \\
\hline V/C & 0.49 & 0.57 & 0.66 & 0.74 & 0.71 & 0.46 & 0.45 & 0.45 & 0.46 & 0.51 \\
\hline \hline No. of link & 11 & 12 & 13 & 14 & 15 & 16 & 17 & 18 & 19 & 20 \\
\hline V/C & 0.44 & 0.43 & 0.36 & 0.46 & 0.44 & 0.44 & 0.46 & 0.70 & 0.73 & 0.65 \\
\hline
\end{tabular}

From the calculate results, it can be seen that Yancheng belt freeway has about $17 \%$ of surplus degrees of capacity in 2012. Many road links are at the service of level 1, operating in good condition. In 2025, with the growth of traffic demand, the belt freeway is about more than $8 \%$ of capacity according to the predicted traffic demand structure. Some road links will exceed Level 3 service standards, and the running condition of belt freeway will decline sharply. Therefore, it is urgent to improve the construction of the expressway system in the urban area by 2025 , and to exert the effect of the urban expressway system on the attraction of entry-exit traffic as well as through traffic, so as to ease the traffic pressure of the belt freeway. 


\section{Conclusions}

In order to overcome the limitation of analysis method of traditional road network capacity, a belt freeway capacity analysis method is put forward in this paper based on reserve capacity of the road network, which is applied to the analysis of the capacity of Yancheng belt freeway on 2012 and 2025. The application results show that the proposed method is not only simple and convenient to use, but also takes into account the actual traffic demand structure, the layout of the road network and the route selection behaviour of the travellers, which can reflect the actual loading situation of belt freeway around the city.

For large cities, in addition to the construction of belt freeway, there should also be a complete expressway system, which, together with belt freeway, constitutes a "high speed road" system in the city area. Both of them are responsible for the organizing function of exit-entry traffic and through traffic in city area. Therefore, the problem which need to be studied further is analysing the capacity of belt freeway based on how to consider the synergistic effect of the expressway system and belt freeway, how to consider their division of labour in the external traffic organization.

\section{Acknowledgement}

This research was financially supported by the united fund of Guizhou department of science and technology and Guizhou normal university (Project No. [2016]7225), and also supported by the fund of the doctoral foundation project of Guizhou normal university on 2015.

\section{References}

1. ZHU Jishuang, ZHANG Ning,Analysis of Factors in Affecting the Loading Capacity of Urban Road Networks. Journal of Transportation Systems Engineering and Information Technology 8,1(2008), pp.92-97 (in Chinese)

2. ZHOU Xizhao,LIU Canqi,YANG Peikun.Timespace Sources of the Network of Urban Road and Space-capacity of Traffic. Journal of Tongji University 24,4(1996), pp. 392- 397 (in Chinese)

3. CHEN Chunmei,REN Futian,RONG Jian,TANG Hui.Application of Random Effective Theory to the Study of the capacity of Road Network. Journal of Beijing Polytechnic University 28,4(2002), pp. 436439 (in Chinese)

4. LI Xuhong,TIAN Feng,GU Zhenghua. Analytical Techniques about the Supply and Demand of Urban Road Network. Journal of Traffic and Transportation Engineering 2,2(2002), pp. 88-90 (in Chinese)

5. Akamatsu,T.,Miyawwaki,O..Maximum Network Capacity Problem under the Transportation Equilibrium Assignment.Infrastrcture Planning Review 12(1995), pp. 719-729
6. Wong S C, Yang H. Reserve capacity of a signal controlled road network. Transportation Research Part B 31,5(1997), pp. 397- 402

7. Hai Yang, Michael G H Bell, Qiang Meng. Modeling the Capacity and Level of Service of Urban Transportation Networks.Transportation Research Part B 34,4(2000), pp. 255- 275

8. XU Liang, GAO Ziyou. Urban Transport Network Design Based on Link Capacity Reliability. China Journal of Highway and Transport 19,2(2006), pp. $86-90$ (in Chinese)

9. ZHU Jishuang, ZHANG Ning. Modeling road network capacity and service level under variable demand pattern. Systems Engineering-Theory \&Practice, 6(2008), pp. 170-176 (in Chinese) 